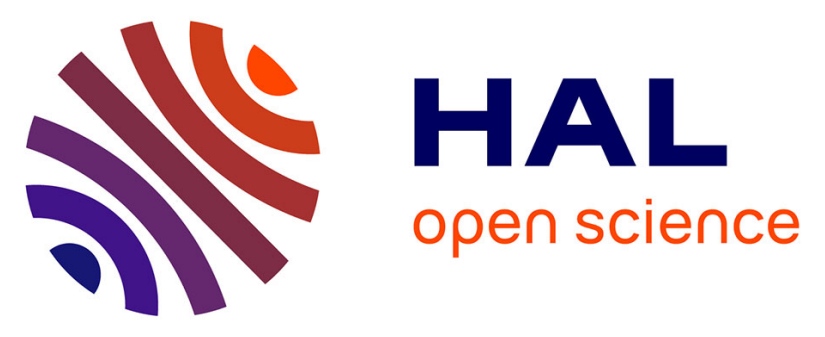

\title{
vLUME: 3D virtual reality for single-molecule localization microscopy
}

\author{
Alexander Spark, Alexandre Kitching, Daniel Esteban-Ferrer, Anoushka \\ Handa, Alexander R Carr, Lisa-Maria Needham, Aleks Ponjavic, Ana Mafalda \\ Santos, James Mccoll, Christophe Leterrier, et al.
}

\section{To cite this version:}

Alexander Spark, Alexandre Kitching, Daniel Esteban-Ferrer, Anoushka Handa, Alexander R Carr, et al.. vLUME: 3D virtual reality for single-molecule localization microscopy. Nature Methods, 2020, 10.1038/s41592-020-0962-1 . hal-02973845

\section{HAL Id: hal-02973845 \\ https://hal.science/hal-02973845}

Submitted on 21 Oct 2020

HAL is a multi-disciplinary open access archive for the deposit and dissemination of scientific research documents, whether they are published or not. The documents may come from teaching and research institutions in France or abroad, or from public or private research centers.
L'archive ouverte pluridisciplinaire HAL, est destinée au dépôt et à la diffusion de documents scientifiques de niveau recherche, publiés ou non, émanant des établissements d'enseignement et de recherche français ou étrangers, des laboratoires publics ou privés. 


\title{
vLUME: 3D virtual reality for single-molecule localization microscopy
}

\author{
Alexander Spark ${ }^{1,8}$, Alexandre Kitching ${ }^{1,8}$, Daniel Esteban-Ferrer $\circledast^{2,8}$, Anoushka Handa ${ }^{2,8}$, \\ Alexander R. Carr' ${ }^{2}$, Lisa-Maria Needham (1) ${ }^{2}$, Aleks Ponjavic ${ }^{3,4}$, Ana Mafalda Santos ${ }^{5}$, James McColl2, \\ Christophe Leterrier ${ }^{6}{ }^{6}$, Simon J. Davis ${ }^{5}$, Ricardo Henriques ${ }^{7,8}$ and Steven F. Lee ${ }^{2 \bowtie}$
}

\begin{abstract}
vLUME is a virtual reality software package designed to render large three-dimensional single-molecule localization microscopy datasets. vLUME features include visualization, segmentation, bespoke analysis of complex local geometries and exporting features. vLUME can perform complex analysis on real three-dimensional biological samples that would otherwise be impossible by using regular flat-screen visualization programs.

Super-resolution microscopy based on three-dimensional single-molecule localization microscopy (3D-SMLM) is now well established $^{1,2}$, and its widespread adoption has led to the development of more than 36 software packages dedicated to quantitative evaluation of the spatial and temporal detection of fluorophore photoswitching. While the initial emphasis in the 3D-SMLM field has clearly been on improving resolution and data quality, there is now a marked absence of $3 \mathrm{D}$ visualization approaches that enable the straightforward, high-fidelity exploration of this type of data. Inspired by the horological phosphorescence points that illuminate watch-faces in the dark, we present $v$ LUME (visualization of the local universe in a micro environment, pronounced 'volume'), an immersive virtual reality (VR)-based visualization software package purposefully designed to render large 3D-SMLM datasets. It is free for academic use. $v$ LUME enables robust visualization, segmentation, annotation and quantification of millions of fluorescence puncta from any $3 \mathrm{D}$-SMLM technique. $v$ LUME has an intuitive user interface and is compatible with all commercial gaming VR hardware (Oculus Rift/Rift S and HTC Vive/Vive Pro; Supplementary Video 1). Although other microscopy data (that is, confocal) visualization tools have previously explored VR technology using volumetric representations $s^{4,5}, v$ LUME has been specifically and purposefully created for SMLM. It accelerates the analysis of highly complex 3D point-cloud data and the rapid identification of defects that are otherwise neglected in global quality metrics. (A comparison with other VR and non-VR tools can be found in Supplementary Table 1.)

$v$ LUME is a point-cloud based 3D-SMLM data visualization tool able to render all pointillism-based multidimensional datasets. It differs from other 3D tools for 3D-SMLM visualization such as $\mathrm{ViSP}^{6}$ by providing a complete VR interactive environment and intuitive interface for life scientists, dedicated to data visualization, segmentation and analysis. Users load multidimensional particle-list datasets into $v$ LUME (.csv files; Fig. 1a), such as those generated by commonly used 3D-SMLM software ${ }^{7,8}$. This allows users to comprehend the spatial and temporal relation between
\end{abstract}

points comprising a 3D structure. In time-lapse data, 3D reconstructions update for each time-point under user control. $v$ LUME is built with the industry-standard cross-platform UnityEngine, providing a high-performance rendering framework that scales with future advances in graphics performance.

$v$ LUME has four key features:

(1) Data exploration and comparison. The configurable user interface allows researchers, without need for programming, to seamlessly switch back and forth from a global view of the entire captured sample to detailed nanoscale views of molecular elements in any arbitrary orientation, faster than with conventional flat screens ${ }^{9}$. Doing so allows the easy local selection of data for further analysis (Supplementary Videos 2 and 3). The software can be used to leverage the human capacity to quickly interpret local features in these data, such as global and local artifacts (Fig. 1b), that are more difficult to trace by automated software without the ground truth being known ${ }^{10}$. In addition, it is easy to quickly evaluate and compare different processing software side by side, such as, for example, QuickPALM versus ThunderSTORM (Fig. 1c). We include example datasets with different sample types, using various SMLM-based super-resolution methods and from different international super-resolution laboratories to demonstrate its broad applicability (Fig. 1b-e and Methods).

(2) Extracting 3D regions of interest (ROI) from complex datasets. Complex biological interactions occur in intricate 3D geometries, with the evaluation of interaction data often requiring the extraction and analysis of specific subselections of a dataset. To demonstrate this capacity of $v$ LUME, we carried out complex segmentation tasks where users needed to identify and select small local features (tens to hundreds of localizations) in data of large dimensions (millions of localizations, Fig. 1d). A single microtubule can be easily extracted from a complex 3D tangle of microtubules within a eukaryotic cell (Fig. 1d, right). This process can be performed in less than 1 min and the ROI exported for further analysis (Methods). Once uploaded, these data subsets can be scaled, highlighted, colored and selected in three dimensions via VR controls (Supplementary Video 4).

(3) Custom analysis of user-defined subregions. Quantitative bioimaging not only relies on high-quality images but quantitative evaluation using bespoke code. Recognizing this, we included a user-definable script interpreter written in the multiparadigm language C\# (Methods). These data can be easily evaluated to

${ }^{1}$ Lume VR Ltd, Oxford, UK. Department of Chemistry, University of Cambridge, Cambridge, UK. ${ }^{3}$ School of Physics and Astronomy, University of Leeds, Leeds, UK. ${ }^{4}$ School of Food Science and Nutrition, University of Leeds, Leeds, UK. ${ }^{5}$ Weatherall Institute of Molecular Medicine, University of Oxford, Oxford, UK. ${ }^{6}$ Aix Marseille Université, CNRS, INP UMR7051, NeuroCyto, Marseille, France. ${ }^{7}$ MRC Laboratory for Molecular Cell Biology, University College London, London, UK. ${ }^{8}$ Instituto Gulbenkian de Ciência, Oeiras, Portugal. ${ }^{9}$ These authors contributed equally: Alexander Spark, Alexandre Kitching, Daniel Esteban-Ferrer, Anoushka Handa. 凶e-mail: sl591@cam.ac.uk 
a

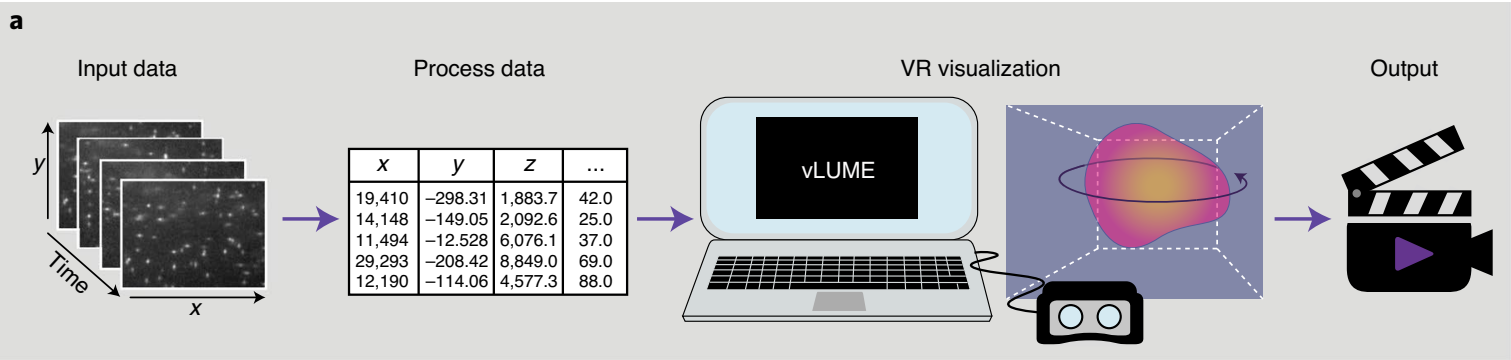

b

Visualize data
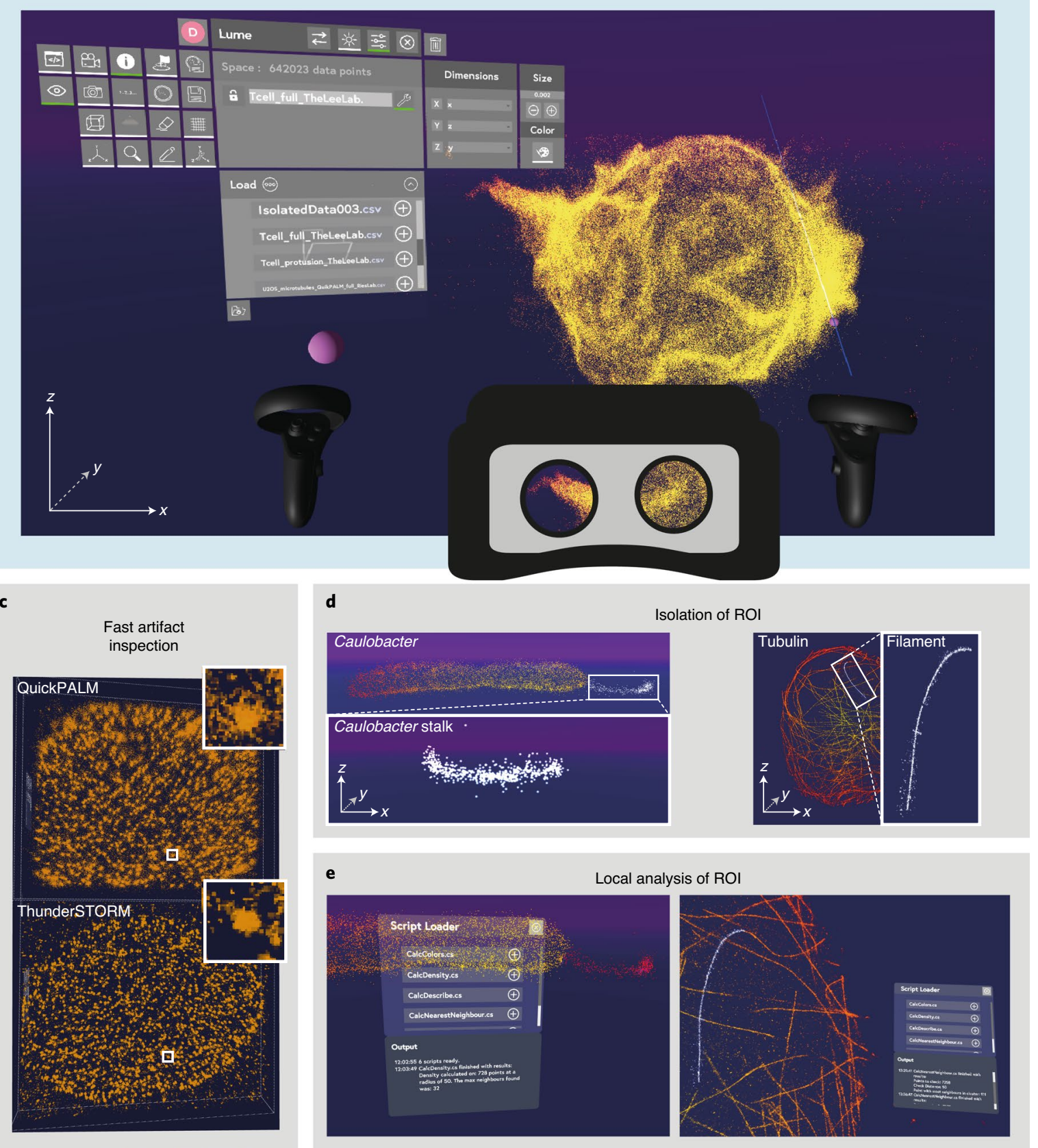

give the user instant quantitative feedback about the specific subregion of their dataset (Fig. 1e). We have included four widely used analyses (Methods section "Scripts in C\# for local analysis"): Ripley's K function ${ }^{11,12}$, nearest neighbor ${ }^{13,14}$ (Supplementary Video 5), local density, and longest and shortest distances. It is our hope to nucleate communities to create, evaluate and share new scripts with each other to enable quantitative SMLM imaging.

(4) Exporting movies for publications and presentations. As well as allowing customization of data for presentation 
Fig. 1 | Features of $v$ LUME. a, $v$ LUME rapidly takes large, multidimensional point-cloud datasets from 2D visualization into an immersive 3D VR environment through a systematic workflow: (1) multidimensional, SMLM image stacks are processed with any standard fitting algorithms providing multiparameter outputs as .csv files. (2) The resultant datasets can then be loaded directly into the vLUME software and instantly visualized in VR. (3) By anchoring at user-defined waypoints around these data, a smoothly interpolated fly-through video can be created and exported, providing the user with a tool to effectively communicate their discoveries. $\mathbf{b}, v L U M E$ facilitates the 3D VR visualization of millions of localizations, demonstrated by the super-resolved membrane of a T cell. The accessible interface enables the user to customize the visual appearance of the localization. The T cell is $\sim 10 \mu m$ in diameter and has an isotropic resolution of $\sim 24 \mathrm{~nm}$ (FSA). c, Comparative inspection of artifacts introduced into data by localization fitting tools can be quickly performed. This is visualized by comparing localizations of nuclear pore complexes fitted with both QuickPALM and ThunderSTORM algorithms. The nuclear pore complex is $\sim 100 \mathrm{~nm}$ in diameter, and the nuclear membrane $\sim 20 \mu \mathrm{m}$ in diameter. Data are taken over a $200 \mathrm{~nm}$ axial range in $z$. d, Selection and isolation of nanoscale, complex biological features can be easily achieved by the user. As examples, the stalk of a Caulobacter crescentus bacterium (left) and a filament of a tubulin network (right) were isolated and a ROI saved for further analysis. The microtubule tangle (right) shows a region of $\sim 20 \times 30 \mu \mathrm{m}$ (and about $500 \mathrm{~nm}$ in depth). The diameter of a single microtubule is $\sim 40 \mathrm{~nm}$. e, The ROI can then be analyzed to instantly quantify desired properties using bespoke C\# scripts (Ripley's K, local density plots, nearest neighbors and any others).

purposes, $v$ LUME also allows custom waypoints (user angle, pitch and yaw) to be defined simply in the VR environment (Fig. 1a) to automatically generate a 'fly-through' video (Supplementary Video 6) to allow researchers to articulate their scientific discoveries.

In summary, $v$ LUME provides a new immersive environment for exploring and analyzing 3D-SMLM data. It enables imaging scientists with any level of expertise to make straightforward analytical sense of what is often highly complex $3 \mathrm{D}$ data. We include a case study of the periodic submembrane scaffold along axons of cultured neurons, made of actin rings regularly spaced every $190 \mathrm{~nm}\left(\right.$ ref. ${ }^{15}$ ). $v$ LUME is used to segment, annotate and analyze complex subregions, including spectrins assembled in the axonal periodic scaffold, submembrane spectrin not organized as rings in cell body and dendrites, cytosolic spectrin, and residual nonspecific labeling outside the cell (Supplementary Video 7). Full documentation and software for $v$ LUME are included in the Methods.

We envisage that users will use $v$ LUME to share and explore existing and new emergent datasets and that the ability to interact with SMLM data in this way may inspire new methods, tools and discoveries. Future directions could include the incorporation of a multi-user tool for numerous users to use $v \mathrm{LUME}$ within the same environment, as well as the incorporation of advanced computation imaging tools such as focused training methods for machine learning.

\section{Online content}

Any methods, additional references, Nature Research reporting summaries, source data, extended data, supplementary information, acknowledgements, peer review information; details of author contributions and competing interests; and statements of data and code availability are available at https://doi.org/10.1038/ s41592-020-0962-1.

Received: 19 January 2020; Accepted: 24 August 2020; Published online: 12 October 2020

\section{References}

1. Von Diezmann, A., Shechtman, Y. \& Moerner, W. E. Three-dimensional localization of single molecules for super-resolution imaging and single-particle tracking. Chem. Rev. 117, 7244-7275 (2017).

2. Lee, M. K., Rai, P., Williams, J., Twieg, R. J. \& Moerner, W. E. Small-molecule labeling of live cell surfaces for three-dimensional super-resolution microscopy. J. Am. Chem. Soc. 136, 14003-14006 (2014).

3. Sage, D. et al. Super-resolution Fight Club: assessment of 2D and 3D single-molecule localization microscopy software. Nat. Methods 16, 387-395 (2019)

4. Theart, R. P., Loos, B. \& Niesler, T. R. Virtual reality assisted microscopy data visualization and colocalization analysis. BMC Bioinf. 18, 64 (2017).

5. Caroline, S. \& Adam, L. H. ConfocalVR: immersive visualization for confocal microscopy. J. Mol. Biol. 430, 4028-4035 (2018).

6. El Beheiry, M. \& Dahan, M. ViSP: representing single-particle localizations in three dimensions. Nat. Methods 10, 689-690 (2013).

7. Henriques, R. et al. QuickPALM: 3D real-time photoactivation nanoscopy image processing in ImageJ. Nat. Methods 7, 339-340 (2010).

8. Ovesný, M., Křížek, P., Borkovec, J., Švindrych, Z. \& Hagen, G. M. ThunderSTORM: a comprehensive imageJ Plug-In for PALM and STORM data analysis and super-resolution imaging. Bioinformatics 30, 2389-2390 (2014).

9. Takashina, T., Ito, M. \& Kokumai, Y. Evaluation of navigation operations in immersive microscopic visualization. VRST' 19 Proc. 68, 68 (2019).

10. Culley, S. et al. Quantitative mapping and minimization of super-resolution optical imaging artifacts. Nat. Methods 15, 263-266 (2018).

11. Lee, S. F., Thompson, M. A., Schwartz, M. A., Shapiro, L. \& Moerner, W. E. Super-resolution Imaging of the nucleoid-associated protein $\mathrm{HU}$ in Caulobacter crescentus. Biophys. J. 100, L31-L33 (2011).

12. Griffié, J. et al. 3D Bayesian cluster analysis of super-resolution data reveals LAT recruitment to the T cell synapse. Sci. Rep. 7, 4077 (2017).

13. Lillemeier, B. F. et al. TCR and Lat are expressed on separate protein islands on $\mathrm{T}$ cell membranes and concatenate during activation. Nat. Immunol. 11, 90-96 (2010).

14. Broadhead, M. J. et al. PSD95 nanoclusters are postsynaptic building blocks in hippocampus circuits. Sci. Rep. 6, 24626 (2016).

15. Leterrier, C., Dubey, P. \& Roy, S. The nano-architecture of the axonal cytoskeleton. Nat. Rev. Neurosci. 18, 713-726 (2017).

Publisher's note Springer Nature remains neutral with regard to jurisdictional claims in published maps and institutional affiliations.

(c) The Author(s), under exclusive licence to Springer Nature America, Inc. 2020 


\section{Methods}

SMLM biological samples included with $v$ LUME. In the installation package of $v$ LUME, we include six biological datasets (12 files) from four different super-resolution microscopy laboratories obtained with a variety of different SMLM methods. The processed datasets (localization files) are saved in the comma separate values (.csv) file format and can be opened as point clouds from the virtual environment of $v$ LUME. They can be found in the IvLume_DatalStreamingAssets folder of the $v$ LUME installation directory (manual).

Plasma membrane of a whole T cell. This shows the large depth-of-focus data of the plasma membrane of a whole Jurkat $\mathrm{T}$ cell imaged in three dimensions using the double helix point spread function (DH-PSF) (imaging done at the Lee laboratory, Cambridge, UK). The cell membrane was labeled using fluorescently tagged wheat germ agglutinin using PAINT, similarly to work previously described ${ }^{16}$. Each cell was imaged as series of $4-\mu \mathrm{m}$ optical slices and reconstructed using overlapping fiducial alignment to create a whole cell of $\sim 10 \mu \mathrm{m}$ with a resolution of $\sim 22 \mathrm{~nm}$ (confirmed by Fourier shell correlation) ${ }^{17}$. Localization data were processed using easy-DH-PSF (10.1038/protex.2013.026). Figure 1b shows the T cell within the virtual environment. The .csv file contains three columns: $x, y$ and $z$ positions (nm) (filename Tcell_full_TheLeeLab.csv).

Microtubules in U-2 OS cells. The microtubules in U-2 OS cells were imaged with astigmatic dSTORM ${ }^{3,18}$ at the Reis laboratory in Heidelberg, Germany. The microtubules were labeled with anti- $\alpha$-tubulin primary and Alexa Fluor 647-tagged secondary antibodies. The single emitters were processed using ThunderSTORM ${ }^{3,8}$ (Fig. 1d,e, right). The comma-delimited file contains five columns: frame number, $x, y$ and $z$ positions (nm) and intensity (photons). These data are openly available (filename U2OS_microtubules_ThunderSTORM_full_RiesLab.csv).

The same raw data were used again (U2OS_microtubules_ThunderSTORM_ full_RiesLab.csv), but processed using QuickPALM ${ }^{3,7}$. The comma-delimited file contains five columns: frame number, $x, y$ and $z$ positions $(\mathrm{nm})$ and intensity (photons) (filename U2OS_microtubules_QuickPALM_full_RiesLab.csv).

A single microtubule obtained from a subset selection from U2OS microtubules_ThunderSTORM_full_RiesLab.csv using $v$ LUME (Fig. 1d, right and Supplementary Video 2). The comma-delimited file contains five columns: frame number, $x$ position, $y$ position and $z$ position $(\mathrm{nm})$ and intensity (photons) (filename U2OS_microtubules_ThunderSTORM_single_RiesLab.csv).

Nuclear pore complexes (NPCs) in U-2 OS cells. The NPC in U-2 OS cells was imaged using astigmatic dSTORM ${ }^{3,18}$ at the Ries laboratory. The NPCs were labeled using NUP107-SNAP-BG tagged with AF647. The single emitters were processed using ThunderSTORM ${ }^{3,8}$ (Fig. 1c, lower). The comma-delimited file contains five columns: frame number, $x, y$ and $z$ positions (nm) and intensity (photons). These data are openly available ${ }^{3}$ (filename U2OS_NPC_ThunderSTORM_RiesLab.csv).

The same raw data for NPCs in U-2 OS cells are shown in U2OS_NPC ThunderSTORM_RiesLab.csv, but were processed using QuickPALM ${ }^{3,7}$ (Fig. 1c, upper). The comma-delimited file contains five columns: frame number, $x$ position, $y$ position and $z$ position (nm) and intensity (photons). These data are openly available ${ }^{3}$ (filename U2OS_NPC_QuickPALM_RiesLab.csv).

Caulobacter crescentus bacteria. C. crescentus bacteria were imaged in three dimensions using the DH-PSF in the Moerner laboratory, Stanford, United States. Colonies of the wild type (CB15N) strain of C. crescentus were grown overnight in a manner previously described ${ }^{2}$ (Fig. $1 \mathrm{~d}$,e left). The bacteria were covalently labeled with $N$-hydroxysuccinimide-functionalized rhodamine spirolactam via a coupling reaction with the exposed amines on the bacteria surface ${ }^{2}$ and imaged in three dimensions using the DH-PSF and processed using bespoke software. The comma-delimited file contains three columns: $x, y$ and $z$ positions (nm) (filename CaulobacterC_2_R09_MoernerLab.csv).

A single $C$. crescentus bacterium showing the stalk, imaged in three dimensions using the DH-PSF. The comma-delimited file contains three columns: $x, y$ and $z$ positions (nm) (CaulobacterC_2_R23_MoernerLab.csv).

Stalk of a C. crescentus bacterium obtained using the data isolation tool in $v$ LUME from dataset CaulobacterC_2_R09_MoernerLab.csv (Supplementary Video 3). The comma-delimited file contains three columns: $x, y$ and $z$ positions (nm) (CaulobacterC_2_R09_stalk_MoernerLab.csv).

Two-channel image of microtubules and clathrin in COS cells. Two-channel images of microtubules and clathrin-coated pits in COS cells were imaged using astigmatism and with DNA-PAINT ${ }^{19}$ at the Leterrier laboratory, Marseille, France. Briefly, cells were labeled with rabbit anti-clathrin light chain and mouse anti- $\alpha$-tubulin antibodies, and secondary antibodies coupled to DNA-PAINT docking strands (Ultivue). The cell was imaged on a commercial N-STORM microscope (Nikon) in buffer containing imaging strands coupled to far-red (clathrin) and red (microtubule) fluorophores by alternating frames. Single emitters were detected using the N-STORM software (Nikon) and processed using ThunderSTORM.

The first channel is labeled for microtubules (anti- $\alpha$-tubulin), imaged in three dimensions (Extended Data Fig. 1, purple). The file contains six columns: frame number, $x, y$ and $z$ positions $(\mathrm{nm})$, lateral uncertainty $(\mathrm{nm})$ and axial uncertainty $(\mathrm{nm})$ (filename COS_microtubules(ch1)_LeterrierLab.csv).

The second channel is labeled for clathrin pits (anti-clathrin light chain), imaged in three dimensions (Extended Data Fig. 1, green). The file contains six columns: frame number, $x, y$ and $z$ positions $(\mathrm{nm})$, lateral uncertainty $(\mathrm{nm})$ and axial uncertainty (nm) (filename COS_clathrin(ch2)_LeterrierLab.csv).

Spectrin scaffold in neurons. Mature neurons (35 d in culture) were fixed and labeled with an anti- $\beta 2$-spectrin primary antibody, then an antimouse secondary antibody coupled to Alexa Fluor 647, and imaged using STORM $^{20}$ at the Leterrier laboratory. Imaging was performed on a commercial N-STORM microscope (Nikon) in Smart Buffer Kit (Abbelight). Single emitters were detected using the N-STORM software (Nikon) and processed using ThunderSTORM. The file contains six columns: frame number, $x, y$ and $z$ positions $(\mathrm{nm})$, lateral uncertainty $(\mathrm{nm})$ and axial uncertainty $(\mathrm{nm})$ (filename Spectrin_neurons_LeterrierLab.csv).

Large datasets visualization. $v$ LUME can easily render large point-cloud datasets in real time (manual). As an example, the T_cell_full_TheLeeLab.csv file has $\sim 650,000$ single-molecule localizations and opens up in $\sim 15$ s on a Gigabyte Aero 15 laptop with an Intel i7 (eighth generation) CPU, an nVIDIA Geforce RTX 2080 GPU and $32 \mathrm{~GB}$ of RAM. It keeps a constant refresh rate of $\sim 80-90 \mathrm{~Hz}$ while navigating the VR environment, which is the industry standard to avoid motion sickness. Roughly 2 million localizations can be rendered without a significant drop in performance using the same system. This performance scales up linearly with the graphics processing power of the system.

\section{Arbitrary selection and isolation of a ROI in large complicated datasets.} Arbitrary selection and isolation of a local ROI is one of the tasks where VR provides an advantage over flat screens thanks to its intuitive navigation ${ }^{9}$. We performed the selection of a stalk in a C. crescentus bacterium and isolated its dataset (sample CaulobacterC_2_R09_stalk_MoernerLab.csv) (Fig. 1d left and Supplementary Video 3) and a single microtubule from a complex tangle (sample U2OS_microtubules_ThunderSTORM_single_RiesLab.csv) (Fig. 1d right and Supplementary Video 2) in less than 1 min (manual). Using the selection feature also enables the application of bespoke C\# scripts to perform analyses in any desired region.

Scripts in C\# for local analysis. An important feature of $v$ LUME is the ability to perform local analysis on a subregion of the point-cloud data (ROI). C\# scripts can be programmed to perform any custom analysis once uploaded to the folder $\backslash$ vLume_Data|StreamingAssets $\backslash$ Scripts within the $v$ LUME installation directory (.cs file extension). Further information can be found on how to program, share and use the scripts as plugins in the next two sections and in the software manual.

We include four useful functions as .cs scripts for the $v$ LUME interpreter that can be used for local cloud data-point analysis and that are often used in SMLM. These can be applied by selecting a ROI and toggling to the scripting menu (see the software manual). Note that, depending on the complexity of the script, a busy indicator may be seen (out of the virtual environment) when the script is called, which is normal. Also, be aware that some operations require more than the available memory, particularly in large datasets (for example, density plots with millions of points that need to compute every single distance from one point to another).

We include four scripts; however, it is our hope that other groups will develop and share their own.

Ripley's K function. Ripley's K function (filename CalcRipleysK.cs) is a spatial point statistics analysis commonly used to evaluate clustering in SMLM data ${ }^{11,12}$. The script computes the Euclidean distance from a series of pairwise points in a ROI and counts the number of neighbors from a single point to a moving $3 \mathrm{D}$ radius (defined by the user and prompted as RegionSize and RegionStep). The input units need to be the same as the dataset. The output of the script is the L function, the linearized and localized Ripley's K function ${ }^{12}$, for every RegionStep from 0 to RegionSize. The script also outputs a .txt file (named after the type of analysis and the time it was performed) in StreamingAssets $\backslash$ Scripts $\backslash$ Output saving the number of points in the ROI, the volume, region size and the step value, then the computed $\mathrm{L}$ (radius), and finally the position of every single point within the ROI.

Note that the function is only taken within the ROI as an isolated region and does not take into account any points in the periphery. Be aware that every single distance from point to point is computed; therefore, large ROIs should be executed only in systems with large amounts of memory. The volume is computed as the smallest bounding box containing the whole dataset.

Nearest-neighbor plot. Nearest-neighbor plot (filename CalcNearestNeighbour.cs) is a widely used analysis tool in super-resolution ${ }^{13,14}$. The user is prompted for the value of the ThresholdRadius (that must be inputted in the same units as the dataset), which will be used to compute the number of neighbors of every single point in the ROI up to the ThresholdRadius. The script then assigns a false color depending on that number. These numbers are normalized with reference to the maximum number of neighbors, red being the lower density and blue the highest 
within a color gradient (note that the colors will be plotted on top of the selection). The script also outputs a .txt file (named after the type of analysis and the time it was performed) in StreamingAssets $\backslash$ Scripts $\backslash$ Output, saving the number of points of the ROI, the radius tested and the number of neighbors of every single point within the ROI together with its position.

As an example, the stalk of the C. crescentus bacterium (dataset CaulobacterC 2 R09_MoernerLab.csv) (Fig. 1e, left, Extended Data Fig. 2) and some NPCs (in the dataset U2OS_NPC_ThunderSTORM_RiesLab.csv) (Supplementary Video 5) were analyzed. For the stalk, we can clearly see that the number is larger in the core and lower in the surface, as expected (Extended Data Fig. 2).

Note that the function is only taken within the ROI as an isolated region and does not take into account any points in the periphery. Be aware that every single distance from point to point is computed; therefore, large ROIs should be executed only in systems with large amounts of memory.

Density. Calculate the density of points in ROI (filename CalcDensity.cs). We provide a very simple implementation that calculates the points within the ROI and divides this value by the volume of the ROI to create the localization density. The result is printed within $v$ LUME as points/volume using the units of the .csv dataset.

Note that the volume is computed as the smallest bounded box that can contain the selected dataset, which is only an approximation for irregular volumes.

Maximum and minimum distances. Calculate the maximum and the minimum distances between points in ROI (filename CalcShortAndFarDistances.cs). For syntax purposes, we provide a very simple script that calculates the maximum and minimum distance between all the points within the ROI. The result is printed within $v$ LUME using the units of the .csv dataset (Supplementary Video 5).

Note that the user should be aware that every single distance from point to point is computed; therefore, large ROIs should be executed only in systems with large amounts of memory.

Collaborating on the open-source C\# plugins using GitHub. $v$ LUME will, hopefully, nucleate communities of SMLM researchers by facilitating active collaboration, development of bespoke analysis features (open-source C\# plugins) and the sharing of these tools through the two GitHub repositories below.

The first GitHub repository houses the base program builds (https://github. com/lumevr/vLume/releases), through which future revisions of $v$ LUME will be released, with updated additional features and bug fixes. The community is able to suggest improvements and inform the developers of any bugs that they may encounter while running $v$ LUME using the default issues report tab in GitHub.

Users can contribute to $v$ LUME by uploading their own open-source code and communicate with other users through the second GitHub repository (https:// github.com/lumevr/vLume_OpenSourcePlugins). In this area, contributors are able to create new branches and request pulls, to develop a subproject that can later be committed and merged to the main branch.

The four original scripts included with the first release of $v$ LUME (see the section "Scripts in C\# for local analysis") can be found in this repository as an initial starting point.

Application programming interface (API) to communicate with $v$ LUME during runtime. The API enables scripts to communicate with the software runtime. It is initialized using the following libraries, which must be included at the start of scripts: using System; using System.Collections.Generic; using System.Collections; using System.Linq; using UnityEngine.

The API is able to perform various functions in two sets of actions: get the manually selected segmented data, and return and push the results back to the VR environment. There are two more actions to view the progress of a script and/or abort it. The number of actions that can be performed can be expanded in future software releases. Please refer to the software manual for a detailed explanation of each of the functions.

Exporting figures and videos. Videos (in .h264 format) and images (in .jpg format) can be captured from the $v$ LUME environment and used for publication or presentation purposes. The fly-through video of the T cell (Supplementary Video 6), the images of the two-channel microtubule-clathrin in COS cells (Extended Data Fig. 1) and the nearest neighbor plot of the isolated C. crescentus (Extended Data Fig. 2) were taken using these integrated features (manual). The .h264 videos can be opened and converted to other formats (that is, .mov or avi for larger compression ratios) in many video players (for example, VLC).

Reporting Summary. Further information on research design is available in the Nature Research Reporting Summary linked to this article.

\section{Data availability}

All the experimental data used in the present communication can be found in the Supplementary Software.

\section{Code availability}

$v$ LUME is available as Supplementary Software. Updated versions of the software can also be found at https://github.com/lumevr/vLume/releases, $v$ LUME software for Windows (with manual, license, samples and scripts). Open-source plugins for $v$ LUME and a forum for collaborative creation and improvement can be found at https://github.com/lumevr/vLume_OpenSourcePlugins.

\section{References}

16. Legant, W. R. et al. High-density three-dimensional localization microscopy across large volumes. Nat. Methods 13, 359-365 (2016).

17. Carr, A. R. Development of Three-Dimensional Super-Resolution Imaging Using a Double-Helix Point Spread Function. Doctoral thesis, Univ. Cambridge (2019); https://doi.org/10.17863/CAM.26413

18. Li, Y. et al. Real-time 3D single-molecule localization using experimental point spread functions. Nat. Methods 15, 367-369 (2018).

19. Jimenez, A., Friedl, K. \& Leterrier, C. About samples, giving examples: optimized procedures for single molecule localization microscopy. Methods 174, 100-114 (2020).

20. Vassilopoulos, S. et al. Ultrastructure of the axonal periodic scaffold reveals a braid-like organization of actin rings. Nat. Commun. 10, 5803 (2019).

\section{Acknowledgements}

We thank M. Lee, J. Yoon and M. Lee from the laboratory of W.E. Moerner (Stanford) for kindly providing the C. crescentus datasets (Fig. 1d,e left). We thank the laboratory of J. Ries (EMBL Heidelberg) for the publicly available NPC data shown in Fig. 1c and for the microtubule datasets shown in Fig. 1d,e, left. We thank F. Boroni-Rueda and K. Friedl for preparation of the neuron samples. D.E.-F. thanks the European Union's Horizon 2020 research and innovation program under the Marie Skłodowska-Curie grant agreement no, 712949 (TECNIOspring Plus) and the Agency for Business Competitiveness of the Government of Catalonia for the research funding leading to these results. A.H. thanks the Engineering and Physical Sciences Research Council (EP/N509620/1). We thank the Royal Society for S.F.L.'s University Research Fellowship (no. UF120277). R.H. was funded by grants from the UK Biotechnology and Biological Sciences Research Council (no. BB/S507532/1), Wellcome Trust (no. 203276/Z/16/Z) and core funding by the MRC Laboratory for Molecular Cell Biology, University College London (grant no. MC_ UU12018/7). We thank The Imagination Group, Imagination Europe and Imagination Labs for their support.

\section{Author contributions}

S.F.L., A.S. and A.K. conceived the initial idea of visualizing 3D SMLM data in VR. A.S. and A.K. wrote, developed and designed the software. A.S, A.H. and D.E.-F. cowrote the open-source C\# scripts. A.H. and D.E.-F. beta-tested the software for SMLM applications. A.H., D.E.-F., L.-M.N., R.H. and S.F.L. cowrote the manuscript. A.R.C. and A.P. performed and analyzed the T cell experiments. A.M.S., S.J.D. and J.M. provided the cell samples and labeling methodology. C.L. provided the spectrin data. All authors edited and revised the manuscript.

\section{Competing interests}

A.S. and A.K. are cofounders of Lume VR Ltd, a company dedicated to creating image analysis software for life sciences and working with groups to tackle visualization and analysis problems. Lume VR Ltd. is providing a free for academic use licence of $v$ LUME governed by Terms of Use that can be found in the Supplementary Software folder. All intellectual property rights in the $v$ LUME software (not the user data), throughout the world, belong to Lume VR Ltd. The remaining authors declare no competing interests.

\section{Additional information}

Extended data is available for this paper at https://doi.org/10.1038/s41592-020-0962-1.

Supplementary information is available for this paper at https://doi.org/10.1038/ s41592-020-0962-1.

Correspondence and requests for materials should be addressed to S.F.L.

Peer review information Rita Strack was the primary editor on this article and managed its editorial process and peer review in collaboration with the rest of the editorial team.

Reprints and permissions information is available at www.nature.com/reprints. 


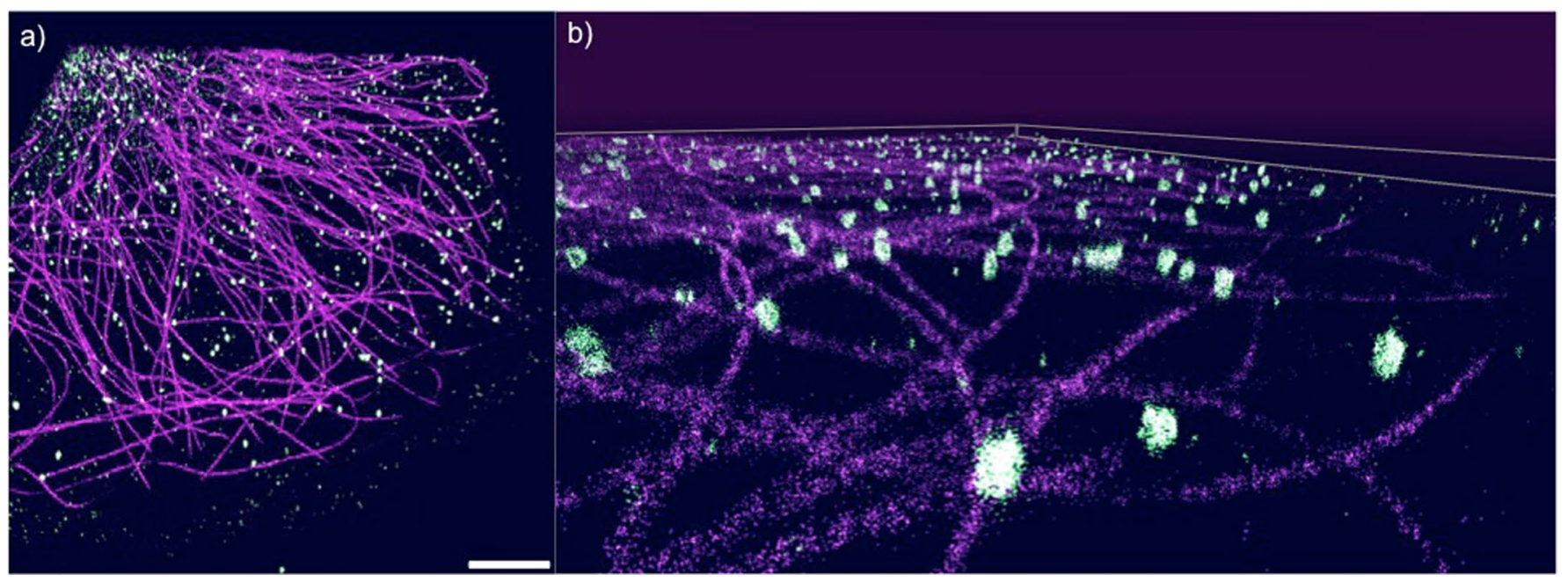

Extended Data Fig. 1 | Microtubules and Clatherin in COS cells. Two-channel data of Microtubules (purple) and Clathrin (green) in COS cells from dataset 5. Scale bar is approximately 1 um. a, A 'birds- eye' projection of the two channels in VLUME. b, The same data set from a different point of view closer to the ground to show the 3D nature of the data. To achieve this superposition the first channel has to be opened in vLUME and the color changed. Then the second channel also needs to be opened and changed in color. Subsequently with simple data translation of one of the datasets the two axes need to be overlapped (this task is very simple in VR, manual). The 3D nature of the oblique figure makes it difficult to render a scale bar. 


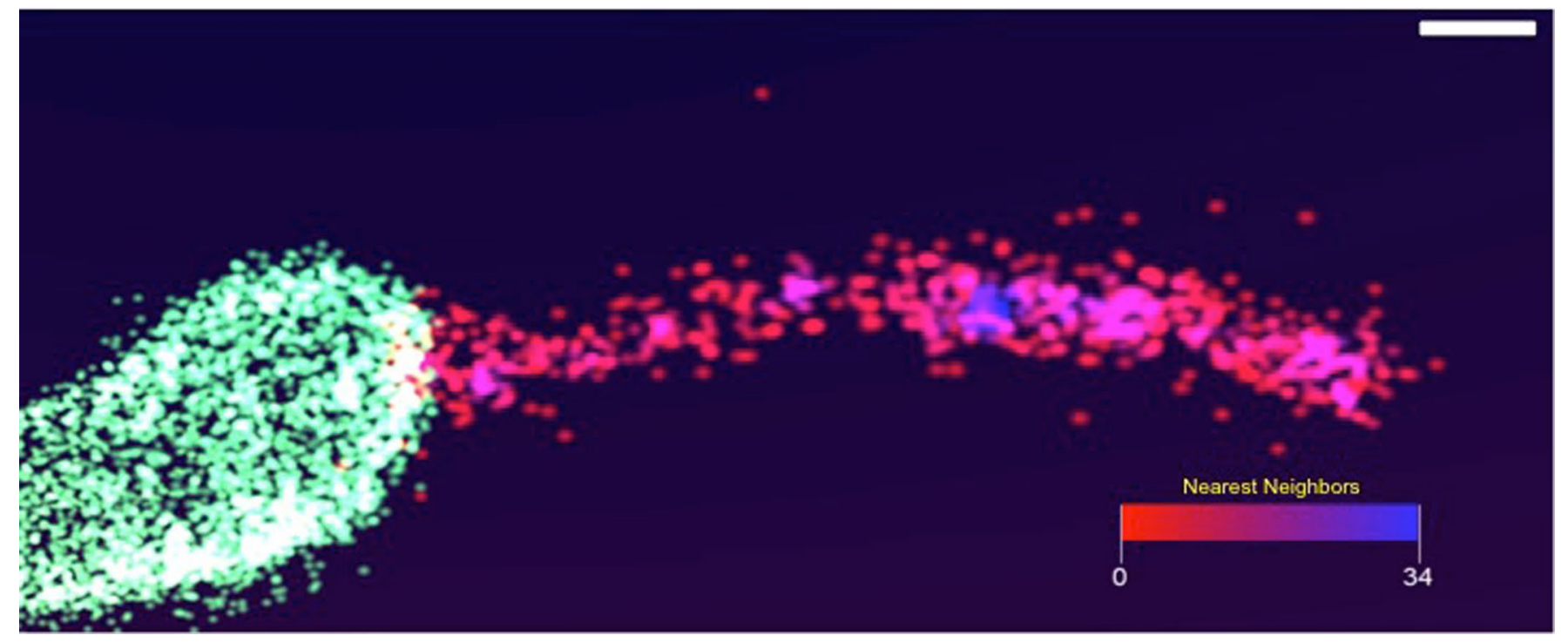

Extended Data Fig. 2 | Nearest Neighbours plot on Caulobacter stalk. Nearest Neighbor plot using the C\# script (CalcNearestNeighbour.cs) after selecting Caulobacter crescentus' stalk from dataset 4 of the Supplementary Information (Fig. 1d, e, left). The red to blue gradient of the image shows an increasing density of nearest neighbors within a radius of $50 \mathrm{~nm}$ (user defined). The color-gradient scale bar goes from 0 to 34 neighbors. Scale bar is approximately $200 \mathrm{~nm}$. 


\section{Reporting Summary}

Nature Research wishes to improve the reproducibility of the work that we publish. This form provides structure for consistency and transparency in reporting. For further information on Nature Research policies, see Authors \& Referees and the Editorial Policy Checklist.

\section{Statistics}

For all statistical analyses, confirm that the following items are present in the figure legend, table legend, main text, or Methods section.

$\mathrm{n} / \mathrm{a}$ Confirmed

\ $\square$ The exact sample size $(n)$ for each experimental group/condition, given as a discrete number and unit of measurement

Х $\square$ A statement on whether measurements were taken from distinct samples or whether the same sample was measured repeatedly

X The statistical test(s) used AND whether they are one- or two-sided

$\triangle \square$ Only common tests should be described solely by name; describe more complex techniques in the Methods section.

$\bigotimes \square$ A description of all covariates tested

Х $\square$ A description of any assumptions or corrections, such as tests of normality and adjustment for multiple comparisons

A full description of the statistical parameters including central tendency (e.g. means) or other basic estimates (e.g. regression coefficient) AND variation (e.g. standard deviation) or associated estimates of uncertainty (e.g. confidence intervals)

$\square$ For null hypothesis testing, the test statistic (e.g. $F, t, r$ ) with confidence intervals, effect sizes, degrees of freedom and $P$ value noted

$\triangle$ Give $P$ values as exact values whenever suitable.

Х For Bayesian analysis, information on the choice of priors and Markov chain Monte Carlo settings

Х $\square$ For hierarchical and complex designs, identification of the appropriate level for tests and full reporting of outcomes

Х $\square$ Estimates of effect sizes (e.g. Cohen's $d$, Pearson's $r$ ), indicating how they were calculated

Our web collection on statistics for biologists contains articles on many of the points above.

\section{Software and code}

Policy information about availability of computer code

Data collection Included sample data is 5 biological data sets, (11 files) from 4 different super-resolution microscopy labs, processed by different localiastions algorithms, all documented in the SI

Data analysis all documented in the SI, we included the fitted localisations

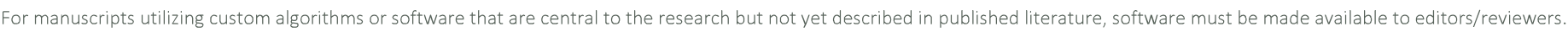
We strongly encourage code deposition in a community repository (e.g. GitHub). See the Nature Research guidelines for submitting code \& software for further information.

\section{Data}

Policy information about availability of data

All manuscripts must include a data availability statement. This statement should provide the following information, where applicable:

- Accession codes, unique identifiers, or web links for publicly available datasets

- A list of figures that have associated raw data

- A description of any restrictions on data availability

\section{Field-specific reporting}

Please select the one below that is the best fit for your research. If you are not sure, read the appropriate sections before making your selection.

\ Life sciences

Behavioural \& social sciences

Ecological, evolutionary \& environmental sciences 


\section{Life sciences study design}

All studies must disclose on these points even when the disclosure is negative.

\begin{tabular}{|c|c|}
\hline Sample size & $\mathrm{n} / \mathrm{a}$ \\
\hline Data exclusions & $\mathrm{n} / \mathrm{a}$ \\
\hline Replication & $n / a$ \\
\hline Randomization & $n / a$ \\
\hline Blinding & $\mathrm{n} / \mathrm{a}$ \\
\hline
\end{tabular}

\section{Reporting for specific materials, systems and methods}

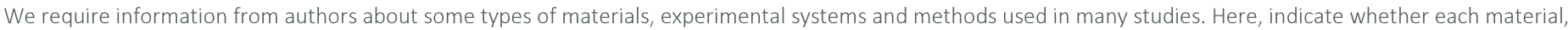

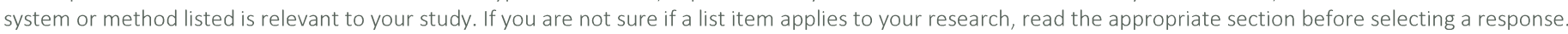

\begin{tabular}{l|lll}
\multicolumn{2}{c}{ Materials \& experimental systems } & & Methods \\
\hline$n / a$ & Involved in the study & & \\
\hline
\end{tabular}

\section{Antibodies}

Antibodies used

Validation all documented in the SI. For dSTORM - DNA-PAINT and STORM labelling strategies cells were labelled with rabbit anti-clathrin and mouse anti-tubulin antibodies, and secondary antibodies coupled to DNA-PAINT docking strands (Ultivue)

Describe the validation of each primary antibody for the species and application, noting any validation statements on the manufacturer's website, relevant citations, antibody profiles in online databases, or data provided in the manuscript.

Eukaryotic cell lines

Policy information about cell lines

Cell line source $(s)$

Authentication

Mycoplasma contamination

Commonly misidentified lines (See ICLAC register) all documented in the SI. Jurkat T-cells, (Created in Davis Lab, Imaged in Lee lab), microtubules and clathrin in COS cells. Microtubules in U-2 OS cells (Ries Lab)

$\mathrm{n} / \mathrm{a}$

No mycoplasma

All cells are used to demonstrate the wide applicabilty of our software platform 